Pacific Journal of Mathematics

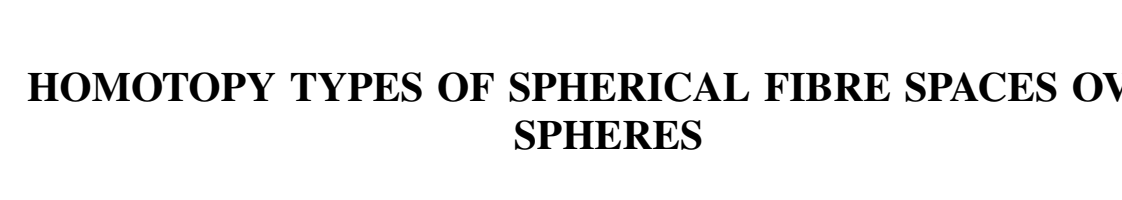




\title{
HOMOTOPY TYPES OF SPHERICAL FIBRE SPACES OVER SPHERES
}

\author{
SEIYA SASAO
}

\begin{abstract}
It is known that the fibre homotopy type of a spherical fibre space over a sphere is determined by its characteristic class. Our purpose is to describe the homotopy type of the total space of a spherical fibre space over a sphere in terms of its characteristic class, and to classify homotopy types of them by defining a kind of equivalence between characteristic classes.
\end{abstract}

I. M. James and J. H. C. Whitehead classified homotopy types of the total space of sphere bundles over spheres in [2] and [3]. Our results are a generalization of their theorems and also an answer to one of problems proposed by J. D. Stascheff in [7]. Let $\mathscr{C}_{k}$ be the space of maps of a $k$-sphere into itself with degree 1 and let $\mathscr{F}_{k}$ be the subspace of $\mathscr{F}_{k}$ consisting of maps preserving the base point ${ }^{t}(0, \cdots, 0,1)$. We denote by $\mathscr{E}_{k, n}(\chi)$ the total space of an orientable $k$-spherical fibre space over an $n$-sphere with $\chi \in \pi_{n-1}\left(\mathscr{G}_{k}\right)$ as its characteristic class. First we shall treat with the case where fibrations have cross-sections. Then we may suppose $\chi=i_{k^{*}}(\xi)$ where $i_{k}: \mathscr{F}_{k} \rightarrow \mathscr{G}_{k}$ denotes the inclusion map.

Now let

$$
\lambda: \pi_{n-1}\left(\mathscr{F}_{k}\right) \longrightarrow \pi_{k+n-1}\left(\mathscr{S}^{k}\right)
$$

be the isomorphism defined by B. Steer in [5]. We are concerned with $\lambda(\xi)$ but not $\chi$.

Then if $i_{k^{*}}(\xi)=i_{k^{*}}\left(\xi^{\prime}\right)$ we claim

$$
\lambda\left(\xi^{\prime}\right)=\lambda(\xi)+\left[x, \iota_{k}\right]
$$

for some $x \in \pi_{n}\left(\mathscr{S}^{k}\right)$ where [,] denotes Whitehead product.

For, let $i$ be the inclusion $\mathscr{R}_{k+1} \rightarrow \mathscr{G}_{k}$ where $\mathscr{R}_{k+1}$ is the rotation group of $\mathscr{S}^{k}$. Clearly $i$ induces a fibre map of the fibration $\mathscr{R}_{k+1} \rightarrow \mathscr{S}^{k}$ into the fibration $\mathscr{G}_{k} \rightarrow \mathscr{S}^{k}$. Since the restriction of $\lambda$ on the image of $\pi_{n \sim 1}\left(\mathscr{R}_{k}\right)$ is equal to (up to sign) ([5]), the homomorphism $\mathscr{J}$ which is defined by G. W. Whitehead in [6], $\lambda$ maps $\partial \pi_{n}\left(\mathscr{S}^{k}\right)$ onto the group $\left[\pi_{n}\left(\mathscr{S}^{k}\right), \iota_{k}\right]$ by the formula $\mathscr{J} \partial(x)=-\left[x, \iota_{k}\right]$ where $\partial$ denotes the boundary homomorphism taken from the homotopy sequences of fibrations. Thus, since $\xi^{\prime}-\xi$ is contained in the $\delta \pi_{n}\left(\mathscr{S}^{k}\right)$, we obtain (1).

Let $\Sigma$ be the natural projection

$$
\pi_{k+n-1}\left(\mathscr{S}^{k}\right) \longrightarrow \pi_{k+n-1}\left(\mathscr{S}^{k}\right) /\left[\pi_{n}\left(\mathscr{S}^{k}\right), \iota_{k}\right]
$$


A map of $\mathscr{S}^{k}$ into itself with degree -1 canonically induces an endmorphism of $\pi_{k+n-1}\left(\mathscr{S}^{k}\right) /\left[\pi_{n}\left(\mathscr{S}^{k}\right), \iota_{k}\right]$. We denote by $\widetilde{\Sigma}$ the composition of $\Sigma$ and the endmorphism. The set

$$
\mathscr{C}\left(\mathscr{E}_{k, n}(\chi)\right)=( \pm \Sigma \lambda(\xi), \pm \widetilde{\Sigma} \lambda(\xi))
$$

is independent from the choice of $\xi$ by (1). Then we shall prove

THEOREM 1. If the fibration $\chi_{i}(i=1,2)$ has a cross-section $(n, k \geqq 2), \mathscr{E}_{k, n}\left(\chi_{1}\right)$ has the same homotopy type as $\mathscr{E}_{k, n}\left(\chi_{2}\right)$ if and only if

(1) if $n \neq k$, or $n=k=$ even $\mathscr{M}\left(\mathscr{E}_{k, n}\left(\chi_{1}\right)\right)=\mathscr{M}\left(\mathscr{E}_{k, n}\left(\chi_{2}\right)\right)$

(2) if $n=k=\operatorname{odd} d \cdot \lambda\left(\xi_{1}\right) \equiv \lambda\left(\xi_{2}\right) \bmod \left[\pi_{n}\left(\mathscr{S}^{k}\right), \iota_{k}\right]$ for some integer $d,(d, m)=1$, where $m$ is the order of $\lambda\left(\xi_{2}\right) \bmod \left[\pi_{n}\left(\mathscr{S}^{k}\right), \iota_{k}\right]$.

If $\mathscr{E}_{k, n}(\chi)$ has the same homotopy type as $\mathscr{S}^{k} \times \mathscr{S}^{n}$ the fibration has a cross-section. Hence we have

CoRollary 1.1. $\quad \mathscr{E}_{k, n}(\chi)$ has the same homotopy type as $\mathscr{S}^{k} \times \mathscr{S}^{n}$ if and only if the fibration $\chi$ is fibre homotopically trivial.

Secondly we consider fibrations which do not necessarily have cross-sections. Therefore, we are mainly concerned in the case $n>k$. However, the case $n=k+1$ is different from others, so we suppose $n \geqq k+2 \geqq 4$.

Let $\bar{\rho}: \mathscr{S}^{k} \rightarrow \mathscr{S}^{k}$ be the homeomorphism defined by

$$
\bar{\rho}\left(x_{1}, x_{2}, \cdots, x_{k+1}\right)=\left(-x_{1}, x_{2}, \cdots, x_{k+1}\right),
$$

and let $\rho: \mathscr{G}_{k} \rightarrow \mathscr{G}_{k}$ be the homeomorphism induced by $\bar{\rho}(\rho(f)=\bar{\rho} f \bar{\rho})$. For any $\alpha \in \pi_{n-1}\left(\mathscr{S}^{k}\right)$, from the diagram

$$
\pi_{k+n-1}\left(\mathscr{S}^{n-1}\right) \underset{\alpha_{*}}{\longrightarrow} \pi_{k+n-1}\left(\mathscr{S}^{k}\right) \underset{\lambda}{\longleftarrow} \pi_{n-1}\left(\mathscr{F}_{k}\right) \underset{i_{k *}}{\longrightarrow} \pi_{n-1}\left(\mathscr{G}_{k}\right),
$$

we have the subgroup of $\pi_{k+n-1}\left(\mathscr{G}_{k}\right)$ defined by

$$
\mathscr{G}(\alpha)=i_{k^{*}} \lambda^{-1} \cdot \alpha_{*} \pi_{k+n-1}\left(\mathscr{S}^{n-1}\right) .
$$

Then we claim

$$
\mathscr{G}(\alpha)=\mathscr{G}(-\alpha) \text { and } \rho_{*}(\mathscr{G}(\alpha))=\mathscr{G}\left(\left(-\iota_{k}\right)_{*} \alpha\right) .
$$

For, the former is clear and the latter follows from the following commutative diagram (see Lemma 2.2)

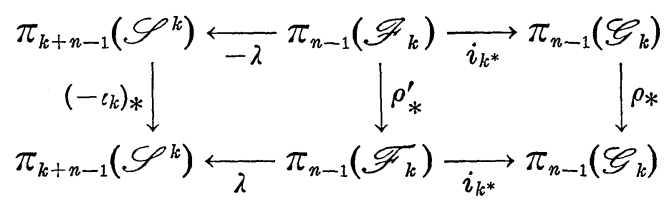


where $\rho \mid \mathscr{F}_{k}=i_{k} \cdot \rho^{\prime}$ is the natural factorization.

Now let $\mathscr{S}[\chi]\left(\chi \in \pi_{n-1}\left(\mathscr{G}_{k}\right)\right)$ be the set of elements

$$
\left\{\chi,-\chi, \rho_{*} \chi,-\rho_{*} \chi\right\}
$$

and let $\mathscr{P}_{k}: \mathscr{G}_{k} \rightarrow \mathscr{S}^{k}$ be the projection of the canonical fibration. We define a relation in $\pi_{n-1}\left(\mathscr{G}_{k}\right)$ as follows $\chi_{1} \sim \chi_{2}$ if and only if $\theta_{1} \equiv \theta_{2} \bmod \mathscr{G}\left(\mathscr{P}_{k}^{*}\left(\theta_{1}\right)\right)$ for some pair $\left(\theta_{1}, \theta_{2}\right), \theta_{i} \in \mathscr{S}\left[\chi_{i}\right]$.

It can be easily checked by (2) that this is an equivalence relation.

THEOREM 2. If $n \geqq k+2 \geqq 4$, then $\mathscr{E}_{k, n}\left(\chi_{1}\right)$ has the same homotopy type as $\mathscr{E}_{k, n}\left(\chi_{2}\right)$ if and only if $\chi_{1} \sim \chi_{2}$.

If fibrations have cross-sections this is an alternative version of Theorem 1. For, since $\mathscr{F}_{k^{*}}\left(\chi_{i}\right)=0$ we have $\chi_{i}=i_{k^{*}}\left(\xi_{i}\right)$. Then the condition $\chi_{1} \sim \chi_{2}$ means that $\chi_{1}= \pm \chi_{2}$ or $\chi_{1}= \pm \rho_{*} \chi_{2}$, i.e.,

$$
i_{k}^{*}\left(\xi_{1}\right)= \pm i_{k^{*}}\left(\xi_{2}\right) \quad \text { or } \quad i_{k^{*}}\left(\xi_{1}\right)= \pm i_{k^{*}}\left(\left(\rho_{*}^{\prime}\right)\left(\xi_{2}\right)\right) \text {. }
$$

These are satisfied if and only if $\xi_{1}= \pm \xi_{2}+\partial \sigma$ or $\xi_{1}= \pm \rho_{*}^{\prime} \xi_{2}+\partial \sigma$ where $\sigma \in \pi_{n}\left(\mathscr{S}^{k}\right)$. Now apply $\lambda$ to the both side, then we have that

$$
\lambda\left(\xi_{1}\right) \equiv \pm \lambda\left(\xi_{2}\right) \quad \text { or } \quad \pm\left(-\iota_{k}\right)_{*} \lambda\left(\xi_{2}\right) \bmod \left[\pi_{n}\left(\mathscr{S}^{k}\right), \iota_{k}\right] \text {. }
$$

This is so if and only if $\mathscr{L}\left(\mathscr{E}_{k, n}\left(\chi_{1}\right)\right)=\mathscr{C}\left(\mathscr{E}_{k, n}\left(\chi_{2}\right)\right)$.

From Theorem 2 the following is easily deduced.

Corollary 2.1. Suppose that $\mathscr{f} \pi_{n-1}\left(\mathscr{R}_{k}\right) \supset \mathscr{P}_{k^{*}}(\chi) \pi_{k+n-1}\left(\mathscr{S}^{n-1}\right)$. If $\mathscr{E}_{k, n}(\chi)(n \geqq k+2 \geqq 4)$ has the same homotopy type as the total space of an orthogonal $\mathscr{S}^{k}$-bundle over $\mathscr{S}^{n}$, then the fibration itself is fibre homotopically equivalent to an orthogonal $\mathscr{S}^{k}$-bundle over $\mathscr{S}_{n}$.

As special cases we have

CoRollary 2.2. Suppose that the fibration $\chi$ has a cross-section. If $\mathscr{E}_{k, n}(\chi)(n \geqq k+2 \geqq 4)$ has the homotopy type of the total space of an orthogonal $\mathscr{S}^{k}$-bundle over $\mathscr{S}^{n}$, the fibration is fibre homotopically equivalent to an orthogonal $\mathscr{S}^{k}$-bundle over $\mathscr{S}^{n}$.

COROLlary 2.3. A k-spherical fibring over $\mathscr{S}^{n}$ is stable fibre homotopically equivalent to an orthogonal $\mathscr{S}^{k}$-bundle over $\mathscr{S}^{n}$ if and only if the total space of the fibring has the same homotopy $\mathscr{S}$-type as the total space of an orthogonal $\mathscr{S}^{k}$-bundle over $\mathscr{S}^{n}$. 
2. $\mathscr{E}_{k, n}(\chi)$ as a $C W$-complex. Let $f:\left(\mathscr{S}^{n-1}, *\right) \rightarrow\left(\mathscr{G}_{k}, 1\right)$ be a representative of $\chi$ and let $\tilde{f}: \mathscr{S}^{n-1} \times \mathscr{S}^{k} \rightarrow \mathscr{S}^{k}$ be the adjoint map. We denote by $\mathscr{K}(f)$ the complex $\mathscr{S}^{k} \cup \mathscr{D}^{n} \times \mathscr{S}^{k}$ obtained from identifying $(x, y)$ with $\widetilde{f}(x, y)$ for $(x, y) \in \mathscr{S}^{n-1} \times \mathscr{S}^{k}$.

Then it is known that $\mathscr{E}_{k, n}(\chi)$ has the same homotopy type as $\mathscr{K}(f)$ (Prop. 1 of [4]). It may be considered that $\mathscr{K}(f)$ is given the natural $C W$-decomposition $\mathscr{S}^{k} \cup e^{n} \cup e^{k+n}$ in which attaching maps for cells are as follows

$$
\begin{gathered}
\alpha: \mathscr{S}^{n-1} \longrightarrow \mathscr{S}^{k}, \alpha(x)=f(x, *) \\
\beta: \mathscr{S}^{k+n-1}=\mathscr{D}^{n} \times \mathscr{S}^{k-1} \cup \mathscr{S}^{n-1} \times \mathscr{D}^{k} \\
\longrightarrow \mathscr{D}^{n} \times * \cup \mathscr{S}^{n-1} \times \mathscr{S}^{k} \underset{\bar{\alpha} \cup \tilde{f}}{\longrightarrow} \mathscr{S}^{k} \cup e^{n}
\end{gathered}
$$

where $\bar{\alpha}:\left(\mathscr{D}^{n}, \mathscr{S}^{n-1}\right) \rightarrow\left(\mathscr{S}^{k} \cup e^{n}, \mathscr{S}^{k}\right)$ denotes the characteristic map for $e^{n}(\alpha=\partial \bar{\alpha})$.

Let $j$ be the inclusion: $\left(\mathscr{S}^{k} \cup e^{n}, *\right) \rightarrow\left(\mathscr{S}^{k} \cup e^{n}, \mathscr{S}^{k}\right)$. Then we have

LEMma 2.1. $\mathscr{P}_{k^{*}}(\chi)=\alpha$, and $j_{*}(\beta)= \pm\left[\bar{\alpha}, \iota_{k}\right]_{r}$ if $n>k+1$ or $\alpha=0$. Thus we can define the orientation of $\mathscr{K}(f)$ by $j_{*}(\beta)=\left[\bar{\alpha}, \iota_{k}\right]_{r}$.

Proof. The former follows from (4) and the definition of $\mathscr{P}_{k^{*}}$. Since the group $\pi_{k+n-1}\left(\mathscr{S}^{k} \cup e^{n}, \mathscr{S}^{k}\right)$ is isomorphic to the direct sum

$$
\mathscr{Z}\left[\bar{\alpha}, \iota_{k}\right]_{r}+\bar{\alpha} \pi_{k+n-1}\left(\mathscr{D}^{n}, \mathscr{S}^{n-1}\right)
$$

under the assumption, $j_{*}(\beta)$ is of the form

$$
m\left[\bar{\alpha}, \iota_{k}\right]_{r}+\bar{\alpha} x
$$

for some integer $m$ and $x \in \pi_{k+n-1}\left(\mathscr{D}^{n}, \mathscr{S}^{n-1}\right)$. Let $\mathscr{X}_{i}(i=k, n, k+n)$ be generators of $\mathscr{H}^{i}(\mathscr{K}(f))=\mathscr{Z}$. Then, by the theorem in [1],

$$
\mathscr{X}_{k} \cup \mathscr{X}_{n}= \pm m \mathscr{X}_{k+n} \text {. }
$$

On the other hand, since $\mathscr{K}^{-}(f)$ has the homotopy type of $\mathscr{E}_{k, n}(\chi)$ we have

$$
\mathscr{X}_{k} \cup \mathscr{X}_{n}= \pm \mathscr{X}_{k+n},
$$

i.e., $m= \pm 1$. And moreover $\bar{\alpha} x=0$ follows from the existence of the projection of the fibration.

Now we consider the special case where $0=\alpha=\mathscr{P}_{k^{*}}(\chi)$. Then the map $f$ may be considered as a map: $\left(\mathscr{S}^{n-1}, *\right) \rightarrow\left(\mathscr{F}_{k}, 1\right)$. Since $\tilde{f} \mid \mathscr{S}^{n-1} \times *=*, \mathscr{S}^{n}$ is naturally imbedded as the image of $\mathscr{D}^{n} \times *$. In this situation, after identifying $\pi_{k+n-1}\left(\mathscr{S}^{k} \vee \mathscr{S}^{n}\right)$ with $\pi_{k+n-1}\left(\mathscr{S}^{k}\right)+$ 
$\pi_{k+n-1}\left(\mathscr{S}^{k} \vee \mathscr{S}^{n}, \mathscr{S}^{k}\right)$, it follows from Lemma 2.1 that

$$
\beta=\iota_{k^{*}}(x)+\left[\iota_{k}, \iota_{n}\right] \text {. }
$$

And also $\beta$ may be considered as follows

$$
\begin{aligned}
\mathscr{S}^{k+n-1}= & \mathscr{D}^{n} \times \mathscr{S}^{k-1} \cup \mathscr{S}^{n-1} \times \mathscr{D}^{k} \underset{1 \times \varphi_{k}}{\longrightarrow} \mathscr{D}^{n} \\
& \times * \cup \mathscr{S}^{n-1} \times \mathscr{S}^{k}{ }_{\varphi_{n} \times * U * \times \tilde{f}} \mathscr{S}^{n} \times * U * \times \mathscr{S}^{k}
\end{aligned}
$$

where $\varphi_{k}$ denotes the identification map: $\mathscr{D}^{k} \rightarrow \mathscr{S}^{k} / \mathscr{\varphi}^{k-1}$.

We make use of $\lambda$ to determine $x$, so we recall the definition of $\lambda$. Let $\varepsilon$ be the map: $\mathscr{S}^{p} \rightarrow \mathscr{F}_{k}$ defined by $\varepsilon()=$ the identity of $\mathscr{S}^{k}$ and let $h$ be a map: $\left(\mathscr{S}^{p}, *\right) \rightarrow\left(\mathscr{F}_{k}, 1\right)$. Since adjoint maps $\tilde{h}, \tilde{\varepsilon}: \mathscr{S}^{p} \times \mathscr{S}^{k} \rightarrow \mathscr{S}^{k}$ has the same restriction on $\mathscr{S}^{p} \vee \mathscr{S}^{k}$, the separation element $d(\tilde{h}, \tilde{\varepsilon}) \in \pi_{p+k}\left(\mathscr{S}^{k}\right)$ is defined. B. Steer defined $\lambda(h)$ by $d(\tilde{h}, \tilde{\varepsilon})$. For example we have (see the diagram (3))

LeMma 2.2. $-\lambda \rho_{*}^{\prime}(\xi)=\left(-\iota_{k}\right)_{*} \lambda(\xi)\left(\xi \in \pi_{p}\left(\mathscr{F}_{k}\right)\right)$.

Proof. Let $g$ be a representative of $\xi$. Then we have

$$
\begin{aligned}
\left(-\iota_{k}\right)_{*} \lambda(\xi) & =\left(-\iota_{k}\right)_{*} d(\widetilde{g}, \tilde{\varepsilon})=\bar{\rho}_{*} d(\widetilde{g}, \tilde{\varepsilon})=d(\bar{\rho} \widetilde{g}, \bar{\rho} \tilde{\varepsilon}) \\
& =d(\bar{\rho} \widetilde{g}, \tilde{\varepsilon}(i d \times \bar{\rho}))=d(\bar{\rho} \widetilde{g}(i d \times \bar{\rho})(i d \times \bar{\rho}), \tilde{\varepsilon}(i d \times \bar{\rho})) \\
& =-d(\bar{\rho} \widetilde{g}(i d \times \bar{\rho}), \tilde{\varepsilon}) .
\end{aligned}
$$

Since $\widetilde{\rho^{\prime} \cdot g}(x, y)=\bar{\rho}\left(\widetilde{g}(x, \bar{\rho}(y))=\bar{\rho}^{\prime} \widetilde{g}(i d \times \bar{\rho})(x, y)\right.$ we have $\widetilde{\rho^{\prime} g}=\bar{\rho} \widetilde{g}(i d \times \bar{\rho})$. Hence $d(\bar{\rho} \widetilde{g}(i d \times \bar{\rho}), \tilde{\varepsilon})=d\left(\widetilde{\rho^{\prime} g}, \tilde{\varepsilon}\right)=\lambda\left(\rho^{\prime} g\right)=\lambda\left(\rho_{*}^{\prime}(\xi)\right)$.

Lemma 2.3. In the expression in (4) we have $x=\lambda(\xi)$, up to sign, where $\xi$ denotes the homotopy class of $f$.

For the proof of Lemma 2.3 we prepare the following general

Lemma 2.4. Let $\mathscr{L}$ be a 1-connected $C W$-complex and let $\mathscr{K}$ be a complex $\mathscr{L} \cup e^{N}(\alpha \sim 0)$. Let $f, g$ be maps: $\mathscr{K} \rightarrow \mathscr{X}$ such that $f|\mathscr{L}=g| \mathscr{L}$ and let $\zeta$ be a map: $\mathscr{S}^{N} \rightarrow \mathscr{K}$ which induces the isomorphism: $\mathscr{H}_{N}\left(\mathscr{S}^{N}, *\right) \rightarrow \mathscr{H}_{N}(\mathscr{K}, \mathscr{L})$. Then we have $d(f, g)=f_{*}(\zeta)$ $-g_{*}(\zeta)$ (up to sign).

Proof. Since $\alpha \sim 0$ there exists a homotopy equivalence $\varphi:(\mathscr{L} \vee$ $\left.\mathscr{S}^{N}, \mathscr{L}\right) \rightarrow(\mathscr{K}, \mathscr{L})$ relative to $\mathscr{L}$. Let $\delta$ be the inclusion $\mathscr{S}^{N} \rightarrow$ $\mathscr{L} \vee \mathscr{S}^{N}$. Then

$$
d(f, g)= \pm d(f \varphi, g \varphi)= \pm\left((f \varphi)_{*} \delta-(g \varphi)_{*} \delta\right) .
$$


From $\varphi^{-1} \zeta \in \pi_{N}\left(\mathscr{L} \vee \mathscr{S}^{N}\right)$ and the assumption on $\zeta$ we have

$$
\varphi_{*}^{-1} \zeta= \pm \delta+\eta\left(\eta \in \pi^{N}(\mathscr{L})\right) \text {, i.e., } \zeta= \pm \varphi_{*}(\delta)+\varphi_{*}(\eta) \text {. }
$$

Hence

$$
\begin{aligned}
f_{*}(\zeta)-g_{*}(\zeta) & =f_{*}\left( \pm \varphi_{*}(\zeta)+\varphi_{*}(\eta)\right)-g_{*}\left( \pm \varphi_{*}(\delta)+\varphi_{*}(\eta)\right) \\
& = \pm\left(f_{*} \varphi_{*}(\delta)-g_{*} \varphi_{*}(\delta)\right)= \pm d(f, g)
\end{aligned}
$$

Proof of Lemma 2.3. Let $Q$ be the identification map:

$$
\mathscr{S}^{n-1} \times \mathscr{S}^{k} \rightarrow \mathscr{S}^{n-1} \times \mathscr{S}^{k} / \mathscr{S}^{n-1} \times * .
$$

The maps

$$
\widetilde{f} \mathscr{Q}^{-1}, \tilde{\varepsilon} \mathscr{Q}^{-1}: \mathscr{S}^{n-1} \times \mathscr{S}^{k} / \mathscr{S}^{n-1} \times * \rightarrow \mathscr{S}^{k}
$$

are well-defined and has the same restriction on $* \times \mathscr{S}^{k} / \mathscr{S}^{n-1} \times *$. The complex $\mathscr{S}^{n-1} \times \mathscr{S}^{k} / \mathscr{S}^{n-1} \times *$ has a form $\mathscr{S}^{k} \cup e^{k+n-1}(\alpha \sim 0)$. Then we apply Lemma 2.4 to the case where

$$
\begin{aligned}
\mathscr{K} & =\mathscr{S}^{n-1} \times \mathscr{S}^{k} / \mathscr{S}^{n-1} \times *, \quad \mathscr{L}=* \times \mathscr{S}^{k} / \mathscr{S}^{n-1} \times *, \\
N & =n+k-1, \quad f=\tilde{f} \mathscr{Q}^{-1}, \quad g=\tilde{\varepsilon} \mathscr{Q}^{-1} \text { and } \mathscr{X}=\mathscr{S}^{k} .
\end{aligned}
$$

Thus we have

$$
\lambda(f)=d(\tilde{f}, \tilde{\varepsilon})=d\left(\widetilde{f} \mathbb{Q}^{-1}, \widetilde{g} \mathbb{Q}^{-1}\right)= \pm\left(\left(f \mathbb{Q}^{-1}\right)_{*}(\zeta)-\left(\tilde{\varepsilon} \mathscr{Q}^{-1}\right)_{*}(\zeta)\right)
$$

for any $\zeta:\left(\mathscr{S}^{k+n-1}, *\right) \rightarrow(\mathscr{K}, \mathscr{L})$ which induces an isomorphism

$$
\zeta_{*}: \mathscr{H}_{k+n-1},\left(\mathscr{S}^{k+n-1}, *\right) \rightarrow \mathscr{H}_{k+n-1}(\mathscr{K}, \mathscr{L}) .
$$

Consider the following commutative diagram

$$
\begin{aligned}
\mathscr{S}^{k+n-1}= & \mathscr{D}^{n} \times \mathscr{S}^{k-1} \cup \mathscr{S}^{n-1} \times \mathscr{D}^{n} \rightarrow \mathscr{D}^{n} \\
& \times * \cup \mathscr{S}^{n-1} \times \mathscr{S}^{k} \underset{* \cup \mathscr{Q}}{\longrightarrow} \mathscr{S}^{n-1} \times \mathscr{S}^{k} / \mathscr{S}^{n-1} \times * \\
& \mid{ }_{\mid} \cup \tilde{f} \mathscr{f}^{-1} \\
& \mathscr{S}^{n} \vee \mathscr{S}^{k} \longrightarrow \mathscr{S}^{k} .
\end{aligned}
$$

Since we can take $\zeta$ with the composition of two maps in the upper row it follows from $\left(\tilde{\varepsilon} \mathbb{Q}^{-1}\right)_{*}(\zeta)=0$ that $\lambda(f)= \pm\left(\tilde{f} \mathbb{Q}^{-1}\right)_{*}(\zeta)$. From the diagram (6) the proof is completed.

3. Proof of Theorem 1. Let $\mathscr{K}$ be a complex of the form

$$
\mathscr{S}^{k} \vee \mathscr{S}^{n} \cup e^{k+n}
$$

where $\beta=\iota_{k} \alpha+\left[\iota_{k}, \iota_{n}\right]$ under the decomposition

$$
\pi_{k+n-1}\left(\mathscr{S}^{k} \vee \mathscr{S}^{n}\right)=\pi_{k+n-1}\left(\mathscr{S}^{k}\right)+\pi_{k+n-1}\left(\mathscr{S}^{n}\right)+\mathscr{Z}\left[\iota_{k}, \iota_{n}\right] .
$$


By the cellular homotopy theorem $\mathscr{K}_{1}$ has the same homotopy type as $\mathscr{K}_{2}$ if and only if there exists a homotopy equivalence $(n, k \geqq 2)$

$$
\Phi: \mathscr{S}^{k} \vee \mathscr{S}^{n} \rightarrow \mathscr{S}^{k} \vee \mathscr{S}^{n}
$$

such that $\Phi_{*}\left(\beta_{1}\right)= \pm \beta_{2}$. Now consider the case $n \neq k$. It is obvious that a map $\Phi$ is homotopy equivalence if and only if $\Phi \mid \mathscr{S}^{k}= \pm \iota_{k}+$ $\iota_{n} \circ \tau\left(\tau \in \pi_{k}\left(\mathscr{S}^{n}\right)\right)$, and $\Phi \mid \mathscr{S}^{n}= \pm \iota_{n}$ if $n<k= \pm \iota_{k}$, and $\Phi \mid \mathscr{S}^{n}=\iota_{k} \circ \sigma+$ $\pm \iota_{n}\left(\sigma \in \pi_{n}\left(\mathscr{S}^{k}\right)\right)$ if $n>k$. From easy computation of $\Phi_{*}\left(\beta_{1}\right)$ we can obtain

Lemma 3.1. If $n \neq k, \mathscr{K}_{1}$ has the same homotopy type as $\mathscr{K}_{2}$ if and only if the set $\left\{ \pm \alpha_{1}, \pm\left(-\iota_{k}\right)_{*} \alpha_{1}\right\}$ is equal to the set

$$
\left\{ \pm \alpha_{2}, \pm\left(\iota_{k}\right)_{*} \alpha_{2}\right\} \bmod \left[\pi_{n}\left(\mathscr{S}^{k}\right), \iota_{k}\right] \text {. }
$$

Next we consider the case $n=k$. By the same way as in [2] we have

Lemma 3.2. (James and Whitehead). If $n=k=$ even, $\mathscr{K}_{1}$ and $\mathscr{K}_{2}$ have the same homotopy type if and only if

$$
\left\{ \pm \alpha_{1}\right\} \equiv\left\{\alpha_{2}\right\} \bmod \left[\pi_{n}\left(\mathscr{S}^{k}\right), \iota_{k}\right] \text {. }
$$

Lemma 3.3. (James and Whitehead). If $n=k=$ odd, $\mathscr{K}_{1}$ and $\mathscr{K}_{2}$ have the same homotopy type if and only if there exists an integer $d$ which is prime to $m_{2}$ and $d \alpha_{1} \equiv \alpha_{2} \bmod \left[\pi_{n}\left(\mathscr{S}^{k}\right), \iota_{k}\right]$ where $m_{2}$ is the order of $\alpha_{2} \bmod \left[\pi_{n}\left(\mathscr{S}^{k}\right), \iota_{k}\right]$.

Thus Theorem 1 follows from Lemmas 3.1, 3.2, 3.3, and 2.3.

4. Some Lemmas. Let $\mathscr{L}$ be a complex of the form $\mathscr{S}^{k} \cup e^{n}$ with the characteristic map $\bar{\alpha}:\left(\mathscr{D}^{n}, \mathscr{S}^{n-1}\right) \rightarrow\left(\mathscr{L}, \mathscr{S}^{k}\right)$ for the $n$-cell. Let $\overline{\mathscr{L}}$ be the complex obtained from identifying $\mathscr{S}^{k}$ of two copies of $\mathscr{L}$, i.e., $\overline{\mathscr{L}}=e^{n} \cup \mathscr{S}^{k} \cup e^{n}$. It may be considered that two maps $\mu_{\imath}(i=1,2): \mathscr{L} \rightarrow \overline{\mathscr{L}}$ and a map $\nu: \overline{\mathscr{L}} \rightarrow \mathscr{L}$ are naturally defined and satisfy $\nu \mu_{i}=$ the identity. Since $\mu_{1}\left|\mathscr{S}^{k}=\mu_{2}\right| \mathscr{S}^{k}$ the separation element $d\left(\mu_{1}, \mu_{2}\right)$ is defined. Then we have

LeMma 4.1. If $\beta \in \pi_{k+n-1}(\mathscr{L})$ and $j_{*}(\beta)=m\left[\bar{\alpha}, \iota_{k}\right]_{r}$, then $\mu_{1^{*}}(\beta)-$ $\mu_{2^{*}}(\beta)=m\left[d\left(\mu_{1}, \mu_{2}\right), \iota_{k}\right]$.

Proof. Consider the following commutative diagram 


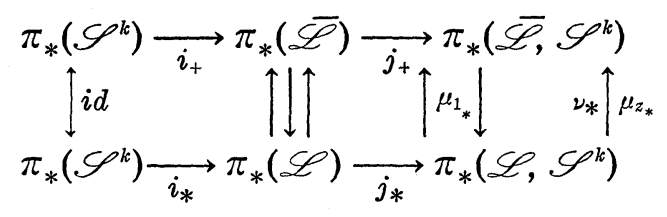

which is taken from the homotopy sequence of the pair and $*=k+$ $n-1$.

From the commutativity it follows that

$$
j_{+}\left(\mu_{1^{*}}(\beta)-\mu_{2^{*}}(\beta)\right)=m\left[\mu_{1^{*}} \bar{\alpha}-\mu_{2^{*}} \bar{\alpha}, \iota_{k}\right]_{r} .
$$

On the orther hand, we have

$$
j_{+}\left[d\left(\mu_{1}, \mu_{2}\right), \iota_{k}\right]=\left[j_{+} d\left(\mu_{1}, \mu_{2}\right), \iota_{k}\right]_{r}=\left[\mu_{1^{*}} \bar{\alpha}-\mu_{2^{+}} \bar{\alpha}, \iota_{k}\right]_{r} .
$$

Thus, for some element $\gamma \in \pi_{*}\left(\mathscr{S}^{k}\right)$, it holds

$$
m\left[d\left(\mu_{1}, \mu_{2}\right), \iota_{k}\right]=\mu_{1^{*}}(\beta)-\mu_{2^{*}}(\beta)+i_{+}(\gamma) .
$$

Applying $\nu_{*}$ to the both side, then, from

$$
\nu_{*} d\left(\mu_{1}, \mu_{2}\right)=d\left(\nu \mu_{1}, \nu \mu_{2}\right)=d(i d, i d)=0 \text { and } \nu \mu_{i}(\beta)=\beta,
$$

we have $\nu_{*} i_{+}(\gamma)=0$. Hence $i_{+}(\gamma)=0$ from the commutativity of the diagram.

As an application of Lemma 4.1 we have

LemMA 4.2. Let $f, g$ be maps: $\mathscr{L} \rightarrow \mathscr{X}$ such that $f|\mathscr{L}=g| \mathscr{L}$. For any $\beta, j_{*}(\beta)=m\left[\bar{\alpha}, \iota_{k}\right]_{r}$, we have

$$
f_{*}(\beta)-g_{*}(\beta)=m\left[d(f, g), f \mid \mathscr{S}^{k}\right] .
$$

Proof. Define a map $f \cup g: \overline{\mathscr{L}} \rightarrow \mathscr{X}$ by

$$
(f \cup g) \mu_{1}=f \text {, and }(f \cup g) \mu_{2}=g \text {. }
$$

Since $d(f, g)=d\left((f \cup g) \mu_{1},(f \cup g) \mu_{2}\right)=(f \cup g)_{*} d\left(\mu_{1}, \mu_{2}\right)$ the proof is completed by applying $(f \cup g)_{*}$ to the both side of the equality in Lemma 4.1.

Let $i d$ be the identity map of $\mathscr{L}(n \geqq k+2 \geqq 4)$ and let $w: \mathscr{L} \rightarrow$ $\mathscr{L}$ be a map with $w\left|\mathscr{S}^{k}=i d\right| \mathscr{S}^{k}$. In general, $d(i d, w)$ is belonging to $\pi_{n}(\mathscr{L})$. However, we have

Lemma 4.3. $w$ is a homotopy equivalence preserving the orientation of the $n$-cell if and only if $d(i d, w)$ is contained in $i_{*} \pi_{n}\left(\mathscr{S}^{k}\right)$. 
Proof. Let $x_{n}, y_{n}$ be the orientation generators of $\mathscr{H}_{n}(\mathscr{L})$, and $\mathscr{H}_{n}\left(\mathscr{S}^{n}\right)$ respectively, and let $\delta$ be $d(i d, w)$. Since $x_{n}-w_{*}\left(x_{n}\right)=$ $\delta_{*}\left(y_{n}\right), x_{n}=w_{*}\left(x_{n}\right)$ holds if and only if $\delta_{*}\left(y_{n}\right)=0$. On the other hand, the diagram

$$
\pi_{n}\left(\mathscr{S}^{k}\right) \underset{i_{*}}{\longrightarrow} \pi_{n}(\mathscr{L}) \longrightarrow \pi_{n}\left(\mathscr{L}, \mathscr{S}^{k}\right)=\mathscr{H}_{n}\left(\mathscr{L}, \mathscr{S}^{k}\right)=\mathscr{H}_{n}(\mathscr{L})
$$

shows that $\delta_{*}\left(x_{n}\right)=0$ is equivalent to $\delta \in i_{*} \pi_{n}\left(\mathscr{S}^{k}\right)$.

Now we prepare lemmas for the proof of Theorem 2. In what follows, we use the notations in $\S 2$ and suppose $n \geqq k+2 \geqq 4$.

LEMma 4.4. Let $i$ be the inclusion: $\mathscr{S}^{k} \rightarrow \mathscr{S}^{k} \cup e^{n} \subset \mathscr{K}(f)$. Then we have

$$
i_{*}^{-1}(0)=\alpha_{*} \pi_{k+n-1}\left(\mathscr{S}^{n-1}\right)
$$

Proof. Since the pair $\left(\mathscr{K}(f), \mathscr{S}^{k}\right)$ is homotopy equivalent to $\left(\mathscr{E}_{k, n}(\chi), \mathscr{S}^{k}\right)$

$$
\pi_{k+n}\left(\mathscr{C}(f), \mathscr{S}^{k}\right)=\pi_{k+n}\left(\mathscr{S}^{n}\right)=E \pi_{k+n-1}\left(\mathscr{S}^{n-1}\right) .
$$

Hence from the homotopy sequence of the triple $\left(\mathscr{K}(f), \mathscr{S}^{k} \cup e^{n}, \mathscr{S}^{k}\right)$ we obtain

$$
\pi_{k+n}\left(\mathscr{S}^{k} \cup e^{n}, \mathscr{S}^{k}\right)=\partial \pi_{k+n+1}\left(\mathscr{K}(f), \mathscr{S}^{k} \cup e^{n}\right) \cup \bar{\alpha}_{*} \pi_{k+n}\left(\mathscr{D}^{n}, \mathscr{S}^{n-1}\right) .
$$

Thus we have that

$$
i_{*}^{-1}(0)=\partial \pi_{k+n}\left(\mathscr{S}^{k} \cup e^{n}, \mathscr{S}^{k}\right)=\alpha_{*} \pi_{k+n-1}\left(\mathscr{S}^{n-1}\right) .
$$

Let $\chi_{i}(i=1,2)$ be elements such that $\mathscr{P}_{k^{*}}\left(\chi_{1}\right)=\mathscr{P}_{k^{*}}\left(\chi_{2}\right)=\alpha$. Then $\beta_{i} \in \pi_{k+n-1}\left(\mathscr{S}^{k} \cup e^{n}\right)$ and there exists an element $\xi \in \pi_{n-1}\left(\mathscr{F}_{k}\right)$ which satisfies $i_{k^{*}}(\xi)=\chi_{1}-\chi_{2}$.

Lemma 4.5. There exists a homotopy equivalence $\varphi: \mathscr{S}^{k} \cup e^{n} \rightarrow$ $\mathscr{S}^{k} \cup e^{n}$ which satisfies

(1) $\varphi_{*}\left(e^{k}\right)=e^{k}, \varphi_{*}\left(e^{n}\right)=e^{n}$

(2) $\beta_{1}-\varphi_{*}\left(\beta_{2}\right)=i_{*} \lambda(\xi)$ (up to sign).

Proof. Let $\kappa: \mathscr{S}^{n} \rightarrow \mathscr{S}_{1}^{n} \vee \mathscr{S}_{2}^{n}$ be a map of type $(1,-1)$ and let $\chi$ be the fibration induced from $\chi_{1} \vee \chi_{2}$ by $\kappa$, i.e., $\chi=\chi_{1}-\chi_{2}$. Since $i_{k^{*}}(\S)=\chi \mathscr{K}(f)$ has the form $\mathscr{S}^{k} \vee \mathscr{S}^{n} \cup e^{k+n}$ by (5). It may be considered that $\kappa$ induces a map $\bar{\kappa}$ :

$$
\begin{aligned}
\mathscr{K}(f) & =\mathscr{S}^{k} \vee \mathscr{S}^{n} \cup e^{k+n} \longrightarrow \mathscr{K}\left(f_{1}\right) \cup \mathscr{K}\left(f_{2}\right) \\
& =\mathscr{D}_{1}^{n} \times \mathscr{S}^{k} \cup \mathscr{S}^{k} \cup \mathscr{D}_{2}^{n} \times \mathscr{S}^{k}
\end{aligned}
$$


which satisfies

$$
\bar{\kappa}_{*}\left(e^{k+n}\right)=e_{1}^{k+n}-e_{2}^{k+n}, \quad \bar{\kappa}_{*}\left(e^{n}\right)=e_{1}^{n}-e_{2}^{n} \text { and } \bar{\kappa}_{*}\left(e^{k}\right)=e^{k} .
$$

Let $\vec{\kappa}: \mathscr{S}^{k} \vee \mathscr{S}^{n} \rightarrow e_{1}^{n} \cup \mathscr{S}^{k} \cup e_{2}^{n}$ be the map obtained from the restriction of $\bar{\kappa}$ on $\mathscr{S}^{k} \vee \mathscr{S}^{n}$ and let $i_{j}$ be the inclusion: $e_{j}^{n} \vee \mathscr{S}^{k} \rightarrow e_{1}^{n} \cup$ $\mathscr{S}^{k} \cup e_{2}^{n}$. Then we have

$$
\overline{\bar{\kappa}}_{*}(\beta)=i_{1^{*}}\left(\beta_{1}\right)-i_{2^{*}}\left(\beta_{2}\right) .
$$

Define the map $r: e_{1}^{n} \cup \mathscr{S}^{k} \cup e_{2}^{n} \rightarrow \mathscr{S}^{k} \cup e^{n}$ by

$$
r \mid e_{1}^{n} \cup \mathscr{S}^{k}=\text { identity }=r \mid \mathscr{S}^{k} \cup e_{2}^{n} .
$$

We claim that

$\left.{ }^{* *}\right) \quad r_{*}(\omega)$ is contained in $\tilde{i}_{*}$-image where $\omega=\overline{\bar{\kappa}} \mid \mathscr{S}^{n}$ and $\tilde{i}$ denotes the inclusion: $\mathscr{S}^{k} \rightarrow e_{1}^{n} \cup \mathscr{S}^{k} \cup e_{2}^{n}$.

For, consider the commutative diagram

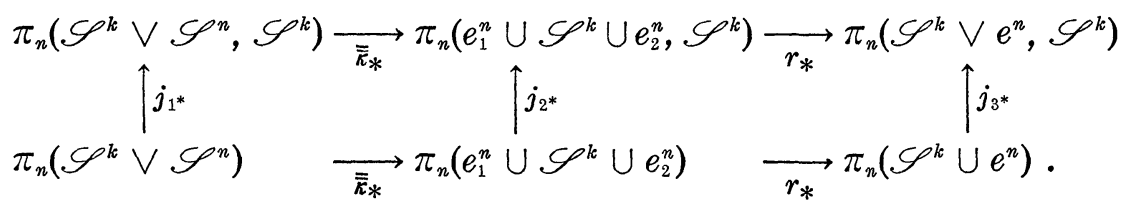

Let $z_{n}$ be the element of $\pi_{n}\left(\mathscr{S}^{k} \vee \mathscr{S}^{n}\right)$ which is represented by $\mathscr{S}^{n}$. Then we have

$$
\begin{aligned}
j_{3^{*}} r_{*}(\omega) & =j_{3^{*}} r_{*} \overline{\bar{\kappa}}\left(z_{n}\right)=r_{*} j_{2^{*}}\left(\overline{\bar{\kappa}}\left(z_{n}\right)\right)=r_{*} \overline{\bar{\kappa}}_{*} j_{1^{*}}\left(z_{n}\right) \\
& =r_{*}\left(i_{1^{*}}\left(\bar{\alpha}_{1}\right)-i_{2^{*}}\left(\bar{\alpha}_{2}\right)\right)=\bar{\alpha}-\bar{\alpha}=0 .
\end{aligned}
$$

Thus $\left({ }^{* *}\right)$ is proved.

Now, by applying $r_{*}$ to the both side of $\left(^{*}\right)$ we have

$$
r_{*} \overline{\bar{\kappa}}_{*}(\beta)=\beta_{1}-\beta_{2} \text {. }
$$

On the other hand, by using (5), we have

$$
\begin{aligned}
r_{*} \overline{\bar{\kappa}}_{*}(\beta) & =r_{*} \overline{\bar{\kappa}}_{*}\left(\iota_{k}( \pm \lambda(\xi))+\left[\iota_{k}, z_{n}\right]\right),\left(\iota_{n}=z_{n}\right) \\
& =i_{*}( \pm \lambda(\xi))+\left[\iota_{k}, r_{*}(\omega)\right] \\
& =i_{*}\left( \pm \lambda(\xi)+\left[\iota_{k}, \omega^{\prime}\right]\right),\left(\omega^{\prime} \in \pi_{n}\left(\mathscr{S}^{k}\right), \tilde{i}_{*} \omega^{\prime}=r_{*}(\omega) \text { by }\left(^{* *}\right)\right) \\
& =i_{*}\left( \pm \lambda(\xi) \pm\left[\omega^{\prime}, \iota_{k}\right]\right)
\end{aligned}
$$

i.e., $\beta_{1}-\beta_{2}=i_{*}\left( \pm \lambda(\xi) \pm\left[\omega^{\prime}, \iota_{k}\right]\right)$.

If we take a map $\varphi: \mathscr{S}^{k} \cup e^{n} \rightarrow \mathscr{S}^{k} \cup e^{n}$ such that $d(i d, \varphi)=\mp \omega^{\prime}$, it follows from Lemma 4.2 and Lemma 2.1 that

$$
\beta_{2}-\varphi_{*}\left(\beta_{2}\right)=i_{*}\left(\mp\left[\omega^{\prime}, \iota_{k}\right]\right) \text { i.e., } \beta_{1}-\varphi_{*}\left(\beta_{2}\right)=i_{*}( \pm \lambda(\xi)) \text {. }
$$

Since $d(i d, \varphi) \in i_{*} \pi_{n}\left(\mathscr{S}^{k}\right) \varphi$ satisfies (1) by Lemma 4.3. 
LEMmA 4.6. There exist homotopy equivalences $u^{\prime}: \mathscr{K}(-f) \rightarrow$ $\mathscr{K}(f)$ and $u^{\prime \prime}: \mathscr{K}(\rho \rho) \rightarrow \mathscr{K}(f)$ which satisfy

(1) $u_{*}^{\prime}\left(e^{k}\right)=e^{k}$ and $u_{*}^{\prime}\left(e^{n}\right)=-e^{n}$,

(2) $u_{*}^{\prime \prime}\left(e^{k}\right)=-e^{k}$ and $u_{*}^{\prime \prime}\left(e^{n}\right)=e^{n}$.

Proof. Let $\mathscr{U}$ be the identification map: $\mathscr{S}^{k}+\mathscr{D}^{n} \times \mathscr{S}^{k} \rightarrow \mathscr{L}(f)$ and define $u^{\prime}, u^{\prime \prime}$ as follows

$$
\begin{gathered}
\rho_{n}\left(x_{1}, x_{2}, \cdots, x_{n}\right)=\left(-x_{1}, x_{2}, \cdots, x_{n}\right),\left(\left(x_{1}, x_{2}, \cdots, x_{n}\right) \in \mathscr{D}^{n}\right) \\
u^{\prime}(x)=x, \quad u^{\prime \prime}(x)=\bar{\rho} x \text { if } x \in \mathscr{S}^{k} \text { and } \\
u^{\prime}(y, z)=\mathscr{U}\left(\rho_{n} y, z\right), \quad u^{\prime \prime}(y, z)=\mathscr{U}(y, \bar{\rho} z) \text { if }(y, z) \in \mathscr{D}^{n} \times \mathscr{S}^{k} .
\end{gathered}
$$

$u^{\prime}$ and $u^{\prime \prime}$ are well-defined by the formulas

$$
-\bar{f}=\bar{f}\left(\left(\rho_{n} \mid \mathscr{S}^{n-1}\right) \times i d\right) \text { and } \tilde{\rho f}=\bar{\rho} \bar{f}(i d \times \bar{\rho}) .
$$

5. Proof of Theorem 2. First of all we prove

Lemma 5.1. If $\mathscr{E}_{k, n}\left(\chi_{1}\right)$ has the same homotopy type as $\mathscr{E}_{k, n}\left(\chi_{2}\right)$ there exists a pair $\left(\theta_{1}, \theta_{2}\right), \theta_{2} \in \mathscr{S}\left[\chi_{i}\right]$ which satisfies

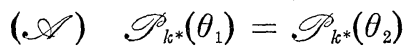

$(\mathscr{B})$ there exists a homotopy equivalence $\psi: \mathscr{K}^{C}\left(g_{1}\right) \rightarrow \mathscr{C}_{C}\left(g_{2}\right)$ with $\psi_{*}\left(e_{1}^{i}\right)=e_{2}^{i}(i=k, n)$

Proof. Let $h: \mathscr{K}\left(f_{1}\right) \rightarrow \mathscr{K}\left(f_{2}\right)$ be a homotopy equivalence which may be considered as a cellular map. Then we have

$$
\mathscr{P}_{k^{*}}\left(\chi_{1}\right)= \pm\left(\mathscr{P}_{k^{*}}\left(\chi_{2}\right)\right) \text { or } \pm\left(-\iota_{k}\right)_{*} \mathscr{P}_{k^{*}}\left(\chi_{2}\right) \text {. }
$$

Since it is clear that each element on the right hand side can be obtained as $\mathscr{P}_{k^{*}}\left(\theta_{2}\right)$ of a suitable $\theta_{2} \in \mathscr{S}\left[\chi_{2}\right]$, there exists a pair $\left(\chi_{1}, \theta_{2}\right)$ which satisfies $(\mathscr{A})$, and a homotopy equivalence $u: \mathscr{K}\left(f_{1}\right) \rightarrow \mathscr{K}\left(g_{2}\right)$ by Lemma 4.6 .

We suppse that $u_{*}\left(e_{1}^{k}\right)=\varepsilon_{k} e_{2}^{k}$ and $u_{*}\left(e_{1}^{n}\right)=\varepsilon_{n} e_{2}^{n} \cdot\left(\varepsilon_{k}, \varepsilon_{n}= \pm 1\right)$. Then we have the equation

$$
\text { (ש) }\left(\varepsilon_{k} \iota_{k}\right)\left(\mathscr{P}_{k^{*}}\left(\chi_{1}\right)=\varepsilon_{n} \mathscr{P}_{k^{*}}\left(\theta_{2}\right)\right. \text {. }
$$

Hence, by $(\mathscr{A})$, we have

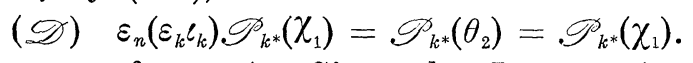

The case of $\varepsilon_{k}=1$. Since, by Lemma 4.6, there exists a homotopy equivalence $u^{\prime}: \mathscr{K}\left(\varepsilon_{n} f_{1}\right) \rightarrow \mathscr{K}\left(f_{1}\right)$ with $u_{*}^{\prime}\left(e^{k}\right)=e^{k}$ and $u_{*}^{\prime}\left(e^{n}\right)=\varepsilon_{n} e^{n}$, the set

$$
\left\{\theta_{1}=\varepsilon_{n} \chi_{1}, \theta_{2}, \psi=u \cdot u^{\prime}\right\}
$$

satisfies $(\mathscr{A})$ and $(\mathscr{B})$.

The case of $\varepsilon_{k}=-1$. Similarly, by Lemma 4.6 , there exists a 
homotopy equivalence $u^{\prime \prime}: \mathscr{K}\left(\varepsilon_{n} \rho f_{1}\right) \rightarrow \mathscr{K}\left(f_{1}\right)$ with $u_{*}^{\prime \prime}\left(e^{k}\right)=-e^{k}$ and $u_{*}^{\prime \prime}\left(e^{n}\right)=e^{n}$. The set

$$
\left\{\theta_{1}=\varepsilon_{n} \rho_{*} \chi_{1}, \theta_{2}, \psi=u \cdot u^{\prime \prime}\right\}
$$

satisfies $(\mathscr{A})$ and $(\mathscr{B})$ by $\mathscr{P}_{k^{*}}\left(\varepsilon_{n} \rho_{*} \chi_{1}\right)=\varepsilon_{n}\left(-\iota_{k}\right) \mathscr{P}_{k^{*}}\left(\chi_{1}\right)$.

Proof of Theorem 2. First we suppose that $\mathscr{E}_{k, n}\left(\chi_{1}\right)$ has the same homotopy type as $\mathscr{E}_{k, n}\left(\chi_{2}\right)$. We choose $\left(\theta_{1}, \theta_{2}, \psi\right)$ as stated in Lemma 5.1. Let $g_{i}$ be a representative of $\theta_{i}$ and let $\gamma_{i}$ be the attaching class for the $(k+n)$-cell of $\mathscr{K}\left(g_{i}\right)$. Let $\varphi: \mathscr{S}^{k} \cup e^{n} \rightarrow \mathscr{S}^{k} \cup e^{n}$ be a map as stated in Lemma $4.5\left(\chi_{i}=\theta_{i}\right)$ and let $\bar{\psi}$ be the map obtained from the restriction of $\psi$ on $\mathscr{S}^{k} \cup e^{n}$. Since $\psi_{*}(\gamma)=\gamma_{2}$ we have

$$
\begin{aligned}
0 & =\gamma_{2}-\bar{\psi}_{*}\left(\gamma_{1}\right) \\
& =\gamma_{2}-\varphi_{*}\left(\gamma_{1}\right)+\varphi_{*}\left(\gamma_{1}\right)-\bar{\psi}_{*}\left(\gamma_{1}\right) \\
& =\left(\gamma_{2}-\varphi_{*}\left(\gamma_{1}\right)\right)+\left[d(\varphi, \bar{\psi}), \iota_{k}\right] \\
& =i_{*}( \pm \lambda(\eta))+\left[d(\varphi, \bar{\psi}), \iota_{k}\right]
\end{aligned}
$$

by Lemma 4.2 and Lemma 2.1 by Lemma 4.5 and

$$
\theta_{2}-\theta_{1}=i_{k^{*}}(\eta) \text {. }
$$

On the other hand, since $d(\varphi, \psi)=d(\varphi, i d)+d(i d, \bar{\psi}), d(\varphi \bar{\psi})$ is contained in $i_{*} \pi_{n}\left(\mathscr{S}^{k}\right)$ by Lemma 4.3. Hence we obtain that

$$
\begin{aligned}
& \lambda(\eta)=\left[\delta, \iota_{k}\right]+i_{*}^{-1}(0) \text { for some } \delta \in \pi_{n}\left(\mathscr{S}^{k}\right) \text { i.e., } \\
& \eta \equiv \lambda^{-1}\left[\delta, \iota_{k}\right] \bmod \lambda^{-1} i_{*}^{-1}(0)=\lambda^{-1} \mathscr{P}_{k^{*}}\left(\theta_{1}\right) \pi_{k+n-1}\left(\mathscr{S}^{n-1}\right)
\end{aligned}
$$

by Lemma 4.4. By applying $i_{k^{*}}$ to the both side we have

$$
\theta_{2}-\theta_{1} \equiv 0 \bmod \mathscr{G}\left(\mathscr{P}_{k^{*}}\left(\theta_{1}\right)\right), \text { i.e., } \chi_{1} \sim \chi_{2} \text {. }
$$

Secondly we assume that $\chi_{1} \sim \chi_{2}$. Hence there exists a pair $\left(\theta_{1}, \theta_{2}\right)$ such that $\theta_{1} \equiv \theta_{2} \bmod \mathscr{G}\left(\mathscr{P}_{k^{*}}\left(\theta_{1}\right)\right)$ which means

$$
\theta_{1}-\theta_{2}=i_{k^{*}}(\eta), \eta \in \pi_{n-1}\left(\mathscr{F}_{k}\right), \lambda(\eta) \in \mathscr{P}_{k^{*}}\left(\theta_{1}\right) \pi_{k+n-1}\left(\mathscr{S}^{n-1}\right) .
$$

Since $\mathscr{P}_{k^{*}}\left(\theta_{1}\right)=\mathscr{P}_{k^{*}}\left(\theta_{2}\right)$ there exists a homotopy equivalence $\varphi: \mathscr{S}^{k} \mathrm{U}$ $e^{n} \rightarrow \mathscr{S}^{k} \cup e^{n}$ which satisfies (see Lemma 4.5)

$$
\gamma_{1}-\varphi_{*}\left(\gamma_{2}\right)=i_{*}( \pm \lambda(\eta)) \text {. }
$$

Since $i_{*}( \pm \lambda(\eta)) \in i_{*} \cdot \mathscr{P}_{k^{*}}\left(\theta_{1}\right) \cdot \pi_{k+n-1}\left(\mathscr{S}^{n-1}\right)=0$ by Lemma 4.4 , we have $\gamma_{1}=\varphi_{*}\left(\gamma_{2}\right)$, i.e., $\varphi$ is extendable over $\mathscr{K}\left(g_{1}\right)$ to $\mathscr{K}\left(g_{2}\right)$. Then, by Lemma $4.6, \mathscr{E}_{k, n}\left(\chi_{1}\right)$ has the same homotopy type as $\mathscr{E}_{k, n}\left(\chi_{2}\right)$.

\section{REFERENCES}

1. I. M. James, Note on cup products, Proc. Amer. Math. Soc., 8 (1957), 374-383.

2. I. M. James and J. H. C. Whitehead, The homotopy theory of sphere bundles over spheres I, Proc. London Math. Soc., (3) 4 (1954), 196-218. 
3. I. M. James and J. H. C. Whitehead, The homotopy theory of sphere bundles over spheres II, Proc. London Math. Soc., (3) 5 (1955), 148-166.

4. J. D. Stasheff, A classification theorem for fibre spaces, Topology, 2 (1963), 239246.

5. B. Steer, Extensions of mappings into H-spaces, Proc. London Math. Soc., (3) 13 (1963), 219-272.

6. G. W. Whitehead, On products in homotopy groups, Ann. of Math., 47 (1946), 460475.

7. $H$-spaces, Neuchâtel (Swisse) Aôut 1970. Lecture notes in Math., Vol. 196, SpringerVerlag.

Received January 11, 1973.

TOKYo Institute of TeChNOLOGY

AND

UNIVERSITY OF OXFORD 



\section{PACIFIC JOURNAL OF MATHEMATICS}

EDITORS

RICHARD ARENS (Managing Editor)

University of California

Los Angeles, California 90024

R. A. Beaumont

University of Washington

Seattle, Washington 98105

J. DugundJI

Department of Mathematics

University of Southern California

Los Angeles, California 90007

D. Gilbarg and J. Milgram

Stanford University

Stanford, California 94305

\section{ASSOCIATE EDITORS}

E. F. BECKENBACH

B. H. NeumanN

F. WOLF

K. YOSHIDA

\section{SUPPORTING INSTITUTIONS}

UNIVERSITY OF BRITISH COLUMBIA CALIFORNIA INSTITUTE OF TECHNOLOGY

UNIVERSITY OF CALIFORNIA

MONTANA STATE UNIVERSITY

UNIVERSITY OF NEVADA

NEW MEXICO STATE UNIVERSITY

OREGON STATE UNIVERSITY

UNIVERSITY OF OREGON

OSAKA UNIVERSITY

\author{
UNIVERSITY OF SOUTHERN CALIFORNIA \\ STANFORD UNIVERSITY \\ UNIVERSITY OF TOKYO \\ UNIVERSITY OF UTAH \\ WASHINGTON STATE UNIVERSITY \\ UNIVERSITY OF WASHINGTON \\ $\stackrel{*}{*} \stackrel{*}{*}{ }^{*}{ }^{*}{ }^{2}$ AMERICAN MATHEMATICAL SOCIETY \\ NAVAL WEAPONS CENTER
}




\section{Pacific Journal of Mathematics}

\section{Vol. 52, No. $1 \quad$ January, 1974}

David R. Adams, On the exceptional sets for spaces of potentials ............ 1

Philip Bacon, Axioms for the Čech cohomology of paracompacta ............ 7

Selwyn Ross Caradus, Perturbation theory for generalized Fredholm operators ..... 11

Kuang-Ho Chen, Phragmén-Lindelöf type theorems for a system of nonhomogeneous equations ............................ 17

Frederick Knowles Dashiell, Jr., Isomorphism problems for the Baire classes .......

M. G. Deshpande and V. K. Deshpande, Rings whose proper homomorphic images are right subdirectly irreducible . . . . . . . . . . . . . . . . . . . . . . . . .

Mary Rodriguez Embry, Self adjoint strictly cyclic operator algebras .............

Paul Erdős, On the distribution of numbers of the form $\sigma(n) / n$ and on some related

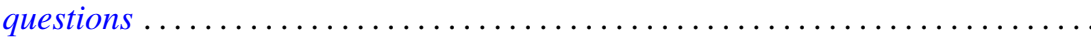

Richard Joseph Fleming and James E. Jamison, Hermitian and adjoint abelian

operators on certain Banach spaces ............................

Stanley P. Gudder and L. Haskins, The center of a poset .................. 85

Richard Howard Herman, Automorphism groups of operator algebras . . . ........

Worthen N. Hunsacker and Somashekhar Amrith Naimpally, Local compactness of families of continuous point-compact relations ....................

Donald Gordon James, On the normal subgroups of integral orthogonal groups ....

Eugene Carlyle Johnsen and Thomas Frederick Storer, Combinatorial structures in

loops. II. Commutative inverse property cyclic neofields of prime-power

order.

Ka-Sing Lau, Extreme operators on Choquet simplexes . . . . . . . . . . . . . . 129

Philip A. Leonard and Kenneth S. Williams, The septic character of 2, 3, 5 and $7 \ldots 143$

Dennis McGavran and Jingyal Pak, On the Nielsen number of a fiber map ........ 149

Stuart Edward Mills, Normed Köthe spaces as intermediate spaces of $L_{1}$ and

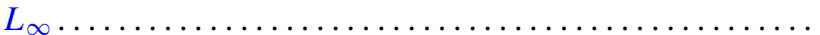

Philip Olin, Free products and elementary equivalence. .

Louis Jackson Ratliff, Jr., Locally quasi-unmixed Noetherian rings and ideals of the principal class.

Seiya Sasao, Homotopy types of spherical fibre spaces over spheres ...

Helga Schirmer, Fixed point sets of polyhedra ...

Kevin James Sharpe, Compatible topologies and continuous irreducible

representations.

Frank Siwiec, On defining a space by a weak base . . . . . . . . . . . . . . . 233

James McLean Sloss, Global reflection for a class of simple closed curves ....... 247

M. V. Subba Rao, On two congruences for primality . .

Raymond D. Terry, Oscillatory properties of a delay differential equation of even

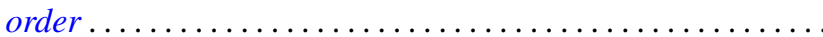

Joseph Dinneen Ward, Chebyshev centers in spaces of continuous functions . .

Robert Breckenridge Warfield, Jr., The uniqueness of elongations of Abelian

groups...

V. M. Warfield, Existence and adjoint theorems for linear stochastic differential

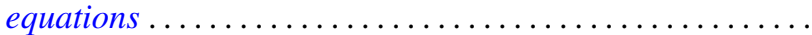

